\section{Sarcoidosis Induced by Tocilizumab: A Paradoxical Event?}

To the Editor:

Tocilizumab (TCZ) is a monoclonal antibody against the interleukin 6 (IL-6) receptor, used in the treatment of rheumatoid arthritis (RA). We describe a case of onset of sarcoidosis under TCZ therapy in a patient with RA.

A 40-year-old woman of North African origin had RA for 12 years. Rheumatoid factor and anticitrullinated antibody were negative. Erosions were absent. Nevertheless, she fulfilled the 2010 American College of Rheumatology/European League Against Rheumatism (ACR/EULAR) RA classification criteria $(7 / 10)$. She was refractory to sulfasalazine and then to methotrexate (MTX). Etanercept was introduced in 2008 and stopped in 2010 because of secondary loss of efficacy. TCZ was initiated in April 2010. The response was good, with the 28-joint Disease Activity Score (DAS28) 0.5 and Health Assessment Questionnaire 0.25 in June 2012, without intolerance. In August 2012, during a stay in Morocco, she developed painful cutaneous nodules, 4 on arms and 2 on legs, with an 8-kg weight loss. Articular symptoms were controlled.

Cutaneous biopsy of arms and legs was performed. Fungal, bacterial, and mycobacterial cultures were negative and histological examination revealed noncaseating epithelioid granulomas in the hypodermis (Figure 1). Tuberculin skin test and QuantiFeron were negative. Angiotensin-converting enzyme was mildly elevated at $70 \mathrm{IU} / 1$ ( $\mathrm{n}=0-68 \mathrm{IU} / \mathrm{l})$. A chest computed tomography showed symmetrical hilar and mediastinal lymph nodes, the largest lymph node measuring $>1 \mathrm{~cm}$ (Figure 2). A left paratracheal and mediastinal lymphadenopathy aspirate biopsy showed 2 noninfective epithelioid granulomas.

The diagnosis of cutaneous and pulmonary sarcoidosis was considered. Infection, including tuberculosis, was ruled out and histological analysis was consistent with sarcoidosis. She did not develop dyspnea, dry cough, kidney involvement, eye involvement, or cardiac lesions.

TCZ was discontinued and RA flared (DAS28 $=5.53$ ). Metacarpophalangeal and interphalangeal joints and knees were swollen and painful. C-reactive protein and erythrocyte sedimentation rates were elevated, 17.0 $\mathrm{mg} / \mathrm{l}$ and $27 \mathrm{~mm}$, respectively. Prednisone was introduced (10 mg/day) with good efficiency on RA and cutaneous nodules. Because of pulmonary involvement, we decided not to restart TCZ and introduced certolizumab with good efficacy and safety.

This patient presented with RA with synovitis and tenosynovitis, without evidence for osteoarticular sarcoidosis. However, the hypothesis of initial sarcoidosis and not RA is possible because of good response to anti-tumor necrosis factor- $\alpha$ (anti-TNF). Nevertheless, it seems less likely because of presence of RA symptoms according to ACR/EULAR criteria and the efficacy of tocilizumab until cessation. To our knowledge, this is the first case of an occurrence of sarcoidosis during TCZ therapy.

It is of interest that sarcoidosis did not appear when the patient was treated with etanercept and, to date, did not flare with certolizumab. Paradoxical effects have been described with TNF blockers, including psoriasis $^{1}$, lupus $^{2}$, vasculitis ${ }^{3}$, and sarcoidosis ${ }^{4}$.

Sarcoidosis is a systemic granulomatous disorder characterized by an accumulation of $\mathrm{T}$ cells and macrophages, activated by various cytokines. We observed a local Th1/Th2 imbalance in favor of Th1-related cytokine expression, like IL-2, interferon gamma, TNF, or IL-6. IL-6 is a macrophage/monocyte-derived cytokine and plays a role in the promotion and maintenance of sarcoid granulomatous inflammation through the activation of CD4+ T cells ${ }^{5}$. In patients with sarcoidosis, IL-6 is increased in bronchoalveolar fluid ${ }^{6}$ and in urine from patients with acute renal failure ${ }^{7}$. IL-6 blockers should have a protective role in sarcoidosis.

Finally, other possible induced events have been reported with TCZ, such as eye inflammation including uveitis ${ }^{8}$, onset of psoriasis ${ }^{9}$, and immune complex-mediated glomerulonephritis ${ }^{10}$.

Our case could represent a new example of paradoxical events with TCZ and suggests the possibility of diseases induced by TCZ therapy.

ANAIZ NUTZ, MD, Hopital Lapeyronie, Rhumatologie; CHARLOTTE PERNET, MD, Hopital Gui de Chauliac, Anatomo-pathologie; BERNARD COMBE, MD, PhD, CHU Lapeyronie, Rhumatologie, Hopital Lapeyronie; JEAN-DAVID COHEN, MD, Hopital Lapeyronie, Rhumatologie, Montpellier, France. Address correspondence to Dr. Cohen, Hopital Lapeyronie, Unité d'Immuno-Rhumatologie, 191 Avenue du Doyen Gaston Giraud, Montpellier 34295, France.

E-mail: jd-cohen@chu-montpellier.fr

\section{REFERENCES}

1. Joyau C, Veyrac G, Dixneuf V, Jolliet P. Anti-tumour necrosis factor alpha therapy and increased risk of de novo psoriasis: is it really a paradoxical side effect? Clin Exp Rheumatol 2012; 30:700-6.

2. Williams VL, Cohen PR. TNF alpha antagonist-induced lupus-like syndrome: report and review of the literature with implications for treatment with alternative TNF alpha antagonists. Int J Dermatol 2011;50:619-25

3. Ramos-Casals M, Britero-Zeron P, Cuadrado MJ, Khamashta MA.

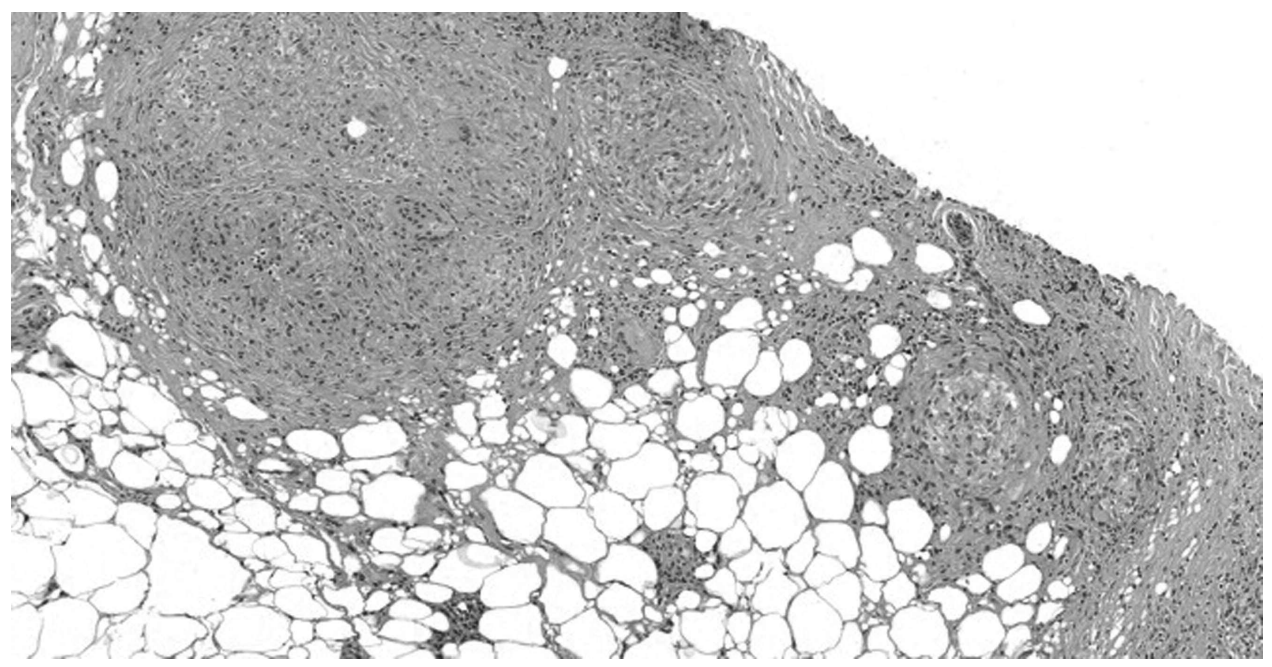

Figure 1. Noncaseating epithelioid granulomas in the hypodermis on cutaneous biopsy. 


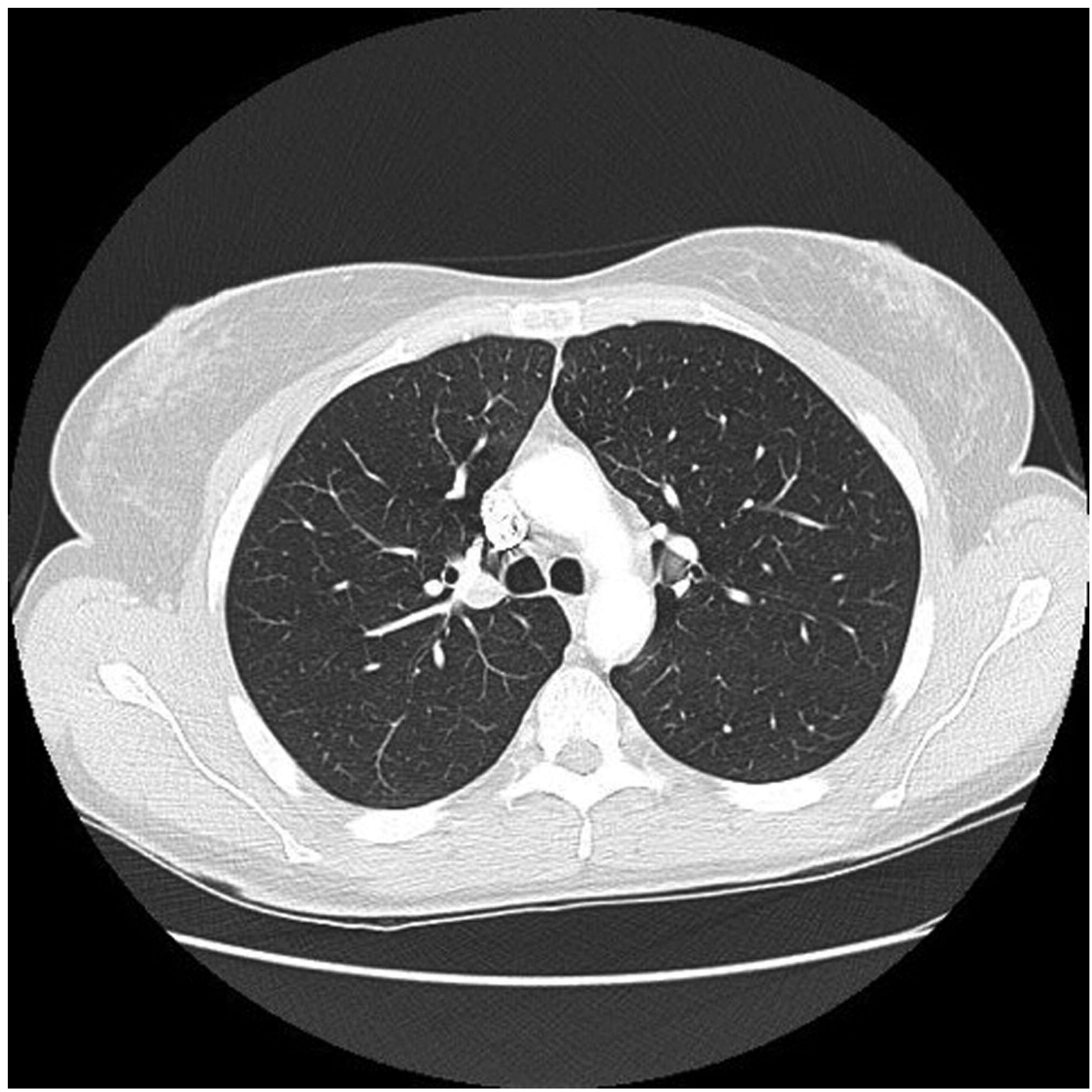

Figure 2. Symmetrical hilar and mediastinal lymph nodes on chest computed tomography.

Vasculitis induced by tumor necrosis factor-targeted therapies. Curr Rheumatol Rep 2008;10:442-8.

4. Daïen Cl, Monnier A, Claudepierre P, Constantin A, Eschard JP, Houvenagel E, et al. Sarcoid-like granulomatosis in patients treated with tumor necrosis factor blockers: 10 cases. Rheumatology 2008;48:883-6.

5. Urbankowski T, Hoser G, Domagala-Kulawik J. Th1/Th2/Th17 related cytokines in the bronchoalveolar lavage fluid of patients with sarcoidosis: association with smoking. Pol Arch Med Wewn 2012;122:320-5.

6. Ishioka S, Saito T, Hiyama K, Haruta Y, Maeda A, Hozawa S, et al Increased expression of tumor necrosis factor-alpha, interleukin-6, platelet-derived growth factor-B and granulocyte-macrophage colony-stimulating factor mRNA in cells of bronchoalveolar lavage fluids from patients with sarcoidosis. Sarcoidosis Vasc Diffuse Lung Disease 1996;13:139-45.
7. Maniwa K, Ogushi F, Haku T, Sone S, Ohmoto Y. Sarcoidosis associated with acute renal failure and increased levels of interleukin-6 in urine. Intern Med 1998:37:757-61.

8. Wendling D, Dernis E, Prati C, Frisch E, Delbosc B. Onset of inflammatory eye disease under tocilizumab treatment for rheumatologic conditions: a paradoxical effect [letter]? J Rheumatol 2011;38:2284

9. Wendling D, Letho-Gyselinck H, Guillot X, Prati C. Psoriasis onset with tocilizumab treatment for rheumatoid arthritis [letter]. J Rheumatol 2012;39:657.

10. Matsuo Y, Mizoguchi F, Kohsaka H, Ito E, Eishi Y, Miyasaka N. Tocilizumab-induced immune complex glomerulonephritis in a patient with rheumatoid arthritis. Rheumatology 2013 Jan 15. [E-pub ahead of print]

J Rheumatol 2013;40:10; doi:10.3899/jrheum.130278 